Dieter Kranz et Friedrich Luft : la critique théâtrale radiophonique dans le Berlin (Est et Ouest) d'après-guerre

Dieter Kranz and Friedrich Luft: Radio theatre criticism in postwar (East and West) Berlin

\title{
Clarisse Cossais
}

\section{(2) OpenEdition}

Journals

\section{Édition électronique}

URL : http://journals.openedition.org/rsl/1209

DOI : $10.4000 /$ rsl. 1209

ISSN : 2271-6246

Éditeur

Éditions Rue d'Ulm

\section{Référence électronique}

Clarisse Cossais, «Dieter Kranz et Friedrich Luft : la critique théâtrale radiophonique dans le Berlin (Est et Ouest)

d'après-guerre », Revue Sciences/Lettres [En ligne], 5 | 2017, mis en ligne le 02 octobre 2017, consulté le 19 avril 2019. URL : http://journals.openedition.org/rsl/1209; DOI : 10.4000/rsl.1209

Ce document a été généré automatiquement le 19 avril 2019

(c) Revue Sciences/Lettres 


\title{
Dieter Kranz et Friedrich Luft : la critique théâtrale radiophonique dans le Berlin (Est et Ouest) d'après-guerre
}

\author{
Dieter Kranz and Friedrich Luft: Radio theatre criticism in postwar (East and \\ West) Berlin
}

Clarisse Cossais

1 Je souhaiterais préciser tout d'abord que c'est en tant que praticienne de la radio publique en Allemagne, où je travaille comme auteure et réalisatrice depuis 1993, que j'interviens dans cet ouvrage. Je vais dans la présente étude évoquer deux critiques de théâtre allemands dont l'enthousiasme et le travail ont servi à collecter la mémoire du théâtre du Berlin d'après-guerre : Friedrich Luft et Dieter Kranz. Mais avant d'entrer dans le vif du sujet, je voudrais aborder quelques considérations générales. La radio est un média encore jeune mais il semble avéré qu'elle a un double rôle dans la problématique qui nous intéresse : elle est à la fois conservatoire mais aussi sélection de la mémoire théâtrale. La sélection de la mémoire théâtrale est liée aux contingences de temps imparti aux programmes consacrés au théâtre. Quand on ne dispose que de quinze minutes parfois de quarante-cinq minutes - par semaine pour rendre compte de l'intense vie théâtrale de sa ville, on est obligé de faire des choix et de mettre de côté nombres de mises en scène qui auraient peut-être elles aussi mérité qu'on les retienne. Ces choix étaient subjectifs, liés à la sensibilité des critiques d'une part ; mais ils étaient également le reflet de l'opposition de deux systèmes idéologiques dont on sait qu'ils prônaient des esthétiques différentes.

2 Cette sélection de la mémoire théâtrale est par ailleurs le résultat parfois fortuit de la pratique concrète de l'archivage des émissions et de circonstances matérielles, telles que l'économie des bandes magnétiques qui, sans aucune logique et parce qu'on n'a pas, en tant qu'auteur de radio, le sentiment de travailler pour l'éternité mais pour une réception 
presque simultanée de ses écrits et de ses propos, a fait disparaitre des trésors. C'est qu'en effet on avait besoin de bandes, et qu'on se servait du matériel plusieurs fois, effaçant ainsi les enregistrements précédents que pour certains nous aimerions tant, aujourd'hui, pouvoir entendre.

D'autre part, Friedrich Luft et Dieter Kranz œuvrant l'un à Berlin-Ouest, l'autre à BerlinEst quelques années plus tard, il est évident que leurs émissions nous renseignent également sur les choix esthétiques pris dans l'une et l'autre partie de la ville. Les thématiques (la dénazification, la reprise économique après-guerre, le monde ouvrier, la division de l'Allemagne), les choix d'auteurs, de mise en scène, la direction des acteurs et même la diction - mais c'est un phénomène plus tardif - varient selon que l'on est d'un côté du mur ou de l'autre. Je n'ai guère la possibilité d'approfondir ces questions dans le cadre de cet ouvrage mais c'est là un vaste chantier de travail qui ne demanderait qu'à être exploré. Enfin, la conception et la pratique de la critique dramatique divergent elles aussi selon que l'on émet depuis Berlin-Ouest ou Berlin-Est, j'y reviendrai ultérieurement.

Friedrich Luft est le premier à mettre en place une émission de critique théâtrale régulière sur la chaîne DIAS (Drahfunk im amerikanischen Sektor - câblodiffuseur dans le secteur américain), qui avait commencé à diffuser en février 1946. À partir du 4 septembre 1946, ce programme diffuse sous le nom RIAS (Radio im amerikanischen Sektor) sur ondes ultra-courtes et ondes moyennes, puis sur grandes ondes à partir de 1954. Né en 1911, Friedrich Luft est entré jeune homme dans l'armée et arrive vers la fin de la guerre dans les services de la section «Film » de l'armée de Terre allemande, où il réalise des films entre autres sur les maladies sexuellement transmissibles ou l'utilisation de masques à gaz lors d'une attaque à la bombe. «Des films, Dieu soit loué, qui ne devaient pas être des films de propagande. Je n'ai jamais été forcé de prêter un faux serment ${ }^{1} »$, commentera-t-il plus tard.

5 Après la fin de la guerre, Berlin est le théâtre des conflits qui opposent les Alliés sur de nombreuses questions. C'est ce qui explique la division de la ville en quatre secteurs et, dès 1946, la création du DIAS où Friedrich Luft passe une audition peu concluante.

Lorsque je sortis, je me rendis bien compte qu'ils étaient contrariés, ils ne voulaient pas de ce que j'avais fait et ils me dirent : «C'est bien beau tout cela, la façon dont vous écrivez, mais vous n'êtes pas du tout compétent pour la radio. Vous comprenez, vous parlez de manière trop rapide, trop énervée. Vous impressionnez les gens au lieu de les intéresser. Nous allons faire lire votre texte par un speaker ou un comédien. " Je trouvais cela ridicule. Ils devaient bien se rendre compte que ma voix a quelque chose de très reconnaissable. Trois jours plus tard, ils me firent revenir ${ }^{2}$.

6 Finalement Friedrich Luft peut proposer, d'abord chaque samedi soir, puis chaque dimanche midi, jusque peu avant sa mort le 24 décembre 1990, ses critiques de quinze minutes qui devinrent rapidement très célèbres, «avec cette même voix qu'on avait voulu autrefois bannir ${ }^{3} »$. L'extrait suivant donne une idée du rapport qu'instaure Luft avec son public:

Bonsoir chers auditeurs, dans les prochaines semaines, nous allons nous retrouver à la même heure, nous allons souvent nous parler et pour cela, il est bon que je me présente : Je m'appelle Luft, Friedrich Luft, je mesure 1,86 m, j'ai les cheveux blond foncé, je pèse $61 \mathrm{~kg}$, j'ai fait des études d'allemand, d'anglais, d'histoire et d'art, je suis né en 1911, je suis obsédé de théâtre et j'aime beaucoup le cinéma. Je bénéficie de la carte d'alimentation catégorie 2 . Et pour finir, je porte, outre mon dernier costume que j'ai pu sauver pendant la guerre, une paire de lunettes en corne. Pourquoi suis-je ici ? Je dois souffrir pour vous ${ }^{4}$. 
7 Cet extrait n'est pas l'original de la première émission de la série Die Stimme der Kritik [La Voix de la critique] du 9 février 1946. Il s'agit d'un enregistrement ultérieur, réalisé en 1956 pour fêter les dix ans de l'émission, et qui a fort heureusement été conservé. Trop rapide, trop énervée, voilà comment ceux qui l'auditionnaient avaient défini le staccato de son débit et sa voix. Diction véloce et essoufflée, phrases souvent interminables alternant avec des phrases d'une extrême brièveté, humour décapant, ton à la fois cultivé et populaire, mélangeant des expressions tirées de divers registres lexicaux : voilà ce qui fait le caractère unique des critiques de Friedrich Luft.

8 Ses émissions Die Stimme der Kritik n’ont été archivées qu’à partir de 1960, par souci d'économie de bandes. Dans le Berlin de l'immédiat après-guerre, où tout était rare et cher, il était impensable de se priver de bandes pour conserver une émission diffusée en direct. Les archives Friedrich-Luft comportent aujourd'hui quatre-cent-huit bandes, dont seule une partie est cataloguée. En revanche, presque tous les manuscrits des émissions sont conservés et l'étude de ces textes est fort intéressante pour nous. Le premier manuscrit conservé est celui du 16 février 1946, six pages tapuscrites très chargées, au revers de pages à l'en-tête de l'Allgemeine Zeitung, un journal pour lequel Luft écrivait également des critiques. Le papier, tout comme les bandes magnétiques, était une denrée rare en 1946. À la lecture de ces textes, on s'aperçoit que Friedrich Luft, dont le débit quasiment incontrôlé pouvait faire penser à une improvisation à partir de quelques notes, rédigeait ses textes par le menu, que tout était écrit. Quand on a vu les manuscrits et saisi leur difficile lisibilité, quand on a à l'oreille la cascade luftienne, on ne peut que saluer l'immense talent d'homme de radio de Friedrich Luft.

9 Son émission compte environ deux milles séquences, elle fonctionna pendant quarantequatre ans selon le même schéma : en entrée, Friedrich Luft consacrait quelques minutes à des considérations personnelles, parlait de rencontres qu'il avait faites, évoquait la situation actuelle. Ensuite il consacrait une longue critique à une pièce dont il chantait les louanges, puis venait une critique de plus petite taille sur une deuxième pièce, et enfin une minuscule critique, quelques lignes seulement, sur une pièce qu'il ne faisait qu'évoquer. Il mélangeait souvent les critiques de théâtre avec celles de cinéma, se permettait parfois quelques excursions à l'opéra, et surtout, il allait, jusqu'à la construction du mur en août 1961, très régulièrement voir des pièces et des films dans le secteur soviétique de la ville. À partir de janvier 1948, Friedrich Luft utilisa pour terminer ses émissions une formule qui allait devenir un proverbe pour les gens de radio en Allemagne: "Gleiche Welle, gleiche Stelle, herzlich auf Wiederhören!" ["Même endroit, même fréquence, cordialement au revoir ! »], traduction insatisfaisante car le verbe hören - écouter - ne s'y retrouve pas. On pourrait lui substituer un jeu de mots : "Même endroit, même fréquence, pour une entente cordiale!" par exemple, ou "à bon entendeur salut!». L'expression figure depuis 2011 sur une plaque commémorative apposée à la façade de Deutschlandradio Kultur sur la Hans-Rosenthal-Platz dans le quartier berlinois de Schöneberg. On l'entend encore dans une émission de 1990, une des dernières que Friedrich Luft anima. La voix du critique est fatiguée, plus de quarante ans ont passé depuis ses débuts à la radio. Dans cette émission, il parle de l'émotion qui l'étreint à voir sa ville réunifiée, et du sentiment qu'il éprouve, celui de se retrouver dans un pays des merveilles trop longtemps interdit.

10 Telle était la forme de l'émission. Quant au contenu, il est foisonnant. La vie culturelle à Berlin dans l'immédiat après-guerre était - contrairement à tous les autres domaines d'une très grande richesse. Luft explique ce foisonnement de représentations 
dramatiques et cinématographiques par le fait que la population était avide de nourritures spirituelles après les douze années passées sous le régime nazi. Je le cite : «Je ne sais pas combien de théâtres existaient. Dans les jardins publics, dans les salles paroissiales, dans les églises. Dans tous les cafés, dans toutes les pièces assez spacieuses, on jouait du théâtre ${ }^{5} \%$. Tandis qu'en parallèle était mis en place le programme de dénazification de la population allemande, les quatre forces alliées rivalisaient entre elles pour montrer aux Allemands vaincus la richesse de leur culture. La population de Berlin profita grandement de cette rivalité. Friedrich Luft commente cet état de fait en disant des officiers des forces d'occupation américaines : «Ce n'est pas à nous qu'ils pensaient, au contraire, ils se disaient: Comment peut-on évincer les Français? On va faire venir Thornton Wilder et lui faire jouer sa pièce, La Peau sur les os [The Skin on our Teeth] ${ }^{6} »$.

Dans son entrée en matière, Luft rendait souvent compte d'anecdotes qu'il avait glanées au cours de la semaine. Il dresse ainsi, sans que ce soit son intention première, un portrait très précis du Berlin d'après-guerre, du champ de ruines que la ville est devenue. Il se sert de son quart d'heure hebdomadaire pour plaider, après douze années de régime nazi, pour

un nouveau public. Nous avons besoin d'un nouveau public, d'un public curieux, d'un public qui participe, d'un public qui ne s'en laisse pas raconter. Qui, par son écoute active, intervient pour ainsi dire dans la scène. Vous ne pouvez vous imaginer ce qu'un tel public peut provoquer et combien il peut changer le théâtre en bien et en $\mathrm{mal}^{7}$.

Il chante les louanges de metteurs en scène qu'il trouve trop peu employés, comme c'est le cas de Jürgen Fehling; il salue le retour sur les planches de comédiens émigrés pendant la guerre, telle Elisabeth Bergner pour qui il éprouvait une immense admiration. Et il constate que le public des zones dites « occidentales » diffère par ses goûts du public de la zone soviétique. La conception du théâtre de Friedrich Luft se résume en un mot: il le conçoit comme un processus actif, dans lequel tous ceux qui participent à la représentation agissent et influencent la création d'un moment éphémère que la radio essaie de conserver, même s'il est évident qu'elle ne peut retenir qu'une petite partie des éléments qui contribuent à l'ensemble. "Le théâtre chante, nous nous en faisons l'écho ${ }^{8}$ " avait-il dit en parlant du rôle de la radio.

Parallèlement à cette émission critique, Die Stimme der Kritik, Friedrich Luft commença à diffuser à partir de 1951 une émission mensuelle, baptisée Wir gehen ins Theater [Nous allons au théâtre], d'environ une heure dans laquelle il exposait des pièces qu'il avait vues. Il présentait les acteurs, le metteur en scène, le scénographe, faisait une introduction à la pièce et laissait passer de très larges extraits, intervenant parfois pour résumer l'action entre deux scènes s'il avait été nécessaire de couper. Les applaudissements finals, auxquels Friedrich Luft n'assistait jamais car il quittait le théâtre dès le premier rideau, servaient de fond sonore à un générique dans lequel il donnait rendez-vous à son public attentif pour une prochaine sortie avec le RIAS.

Les forces d'occupation soviétiques avaient, dès mai 1945, créé la station de radio Berliner Rundfunk [Radio Berlin] dont les locaux se trouvaient en zone britannique. Les tensions devenant de plus en plus grandes entre les forces alliées, avec un point culminant lors du blocus de Berlin-Ouest en 1948, elles décidèrent d'installer le Berliner Rundfunk dans leur zone, à König-Wusterhausen, puis de fonder une maison de la radio nationale. À partir de ce moment, et sans que cela soit officiel, il fut mal considéré en RDA d'écouter les médias ouest-allemands et en particulier le RIAS. On installa même des brouilleurs d'ondes pour 
empêcher que le programme du RIAS soit capté. C'est au Berliner Rundfunk qu'à partir de 1956, l'autre critique de théâtre et d'opéra que j'aimerais évoquer dans cette étude, proposa une émission hebdomadaire d'environ quinze minutes intitulée Atelier und Bühne [Atelier et Scène], qui rendait souvent compte de mises en scène ayant eu lieu non seulement à Berlin mais aussi dans des théâtres des grandes villes de la RDA.

Dieter Kranz est né en 1934 à Berlin, dans une famille modeste. Il a fait des études pour devenir comédien à Weimar, puis des études de littérature allemande à Leipzig. Il commence à travailler au Berliner Rundfunk en 1956, en tant que « rédacteur », ce qui est l'équivalent du « producteur » dans le domaine de la radio en France. Les émissions Atelier und Bühne n'avaient apparemment pas été conservées: j'ai eu accès aux manuscrits, archivés à l'Académie des arts de Berlin, mais les bandes n'ont pas encore refait surface. Si j'emploie cette expression, c'est que l'Académie des Arts de Berlin a en effet reçu trente-sept cartons remplis de bandes qui constituent le fonds sonore des archives de Dieter Kranz. Elles étaient restées des années dans un garage. Ces bandes ont été nettoyées mais n'ont pas encore été répertoriées, et ce travail s'annonce très laborieux dans la mesure où les cartons ne disposent de quasiment aucune information concernant leur contenu.

Ce qui est bien conservé en revanche - même si ce n'est pas dans son intégralité -, c'est la série des émissions que Dieter Kranz anima chaque mois de 1961 à 1991 pour le Berliner Rundfunk et qui s'appelait Berlin, Weltstadt des Theaters [Berlin, capitale mondiale du théâtre]. Les bandes se trouvent aux Archives nationales de la radio situées à Potsdam Babelsberg, elles ne sont qu'en partie digitalisées. La première émission par exemple, dans laquelle l'auteur se livre volontiers à des confidences précieuses sur son projet et sa manière de concevoir son travail, n'a malheureusement pas été conservée. L'émission était de durée variable en fonction des sujets qu'elle traitait, entre quarante-cinq et soixante minutes. Dieter Kranz "guidait» les auditeurs dans l'émission, selon l'expression consacrée. Parfois aussi, s'il le pensait nécessaire, il confiait ce rôle à un collègue, voire, dans quelques cas assez isolés, à un metteur en scène qui parlait du spectacle dont on entendait des extraits.

Ainsi, dans le $216^{e}$ épisode de l'émission Berlin, Weltstadt des Theaters, Kranz présente un rapide passage de la pièce de Goldoni, Arlequin, serviteur de deux maitres, mise en scène par Giorgio Strehler au Piccolo Teatro de Milan. Il intervient dans l'extrait pour venir en aide aux auditeurs qui ne comprennent pas l'italien : il décrit la scène dans laquelle Arlequin s'adresse à ses deux maîtres et parle de la musicalité des dialogues et des réactions animées du public, qui rit à gorge déployée. Vient ensuite un long entretien en allemand avec Giorgio Strehler :

Giorgio STREHLER : Bien sûr, je rencontre de gros problèmes, le théâtre que je fais n'est pas un théâtre confortable, j'ai toujours cru qu'on pouvait changer le monde avec le théâtre, pas seulement avec lui, mais aussi avec le théâtre et qu'on pouvait contribuer à changer la société ${ }^{\text {. }}$

18 Tandis que Friedrich Luft entretenait volontiers le culte qui était fait autour de sa personne, Dieter Kranz brille par sa modestie. Sa diction est douce, lente, posée, il formule des questions précises, est toujours très curieux et pointu dans ses interrogations, mais on sent bien aussi qu'il ne fait pas partie de ceux qui aiment s'entendre parler. Devenu en 1991 membre du jury du prix des Critiques de disques, il définit son rôle de la manière suivante : 
Dans mon métier, je n'ai jamais perçu ma fonction comme celle d'un arbitre qui a toujours raison mais comme celle d'un passionné de musique et de théâtre qui aimerait transmettre son amour de l'art au public. Persuadé qu'il ne peut jamais y avoir une seule interprétation valable, j'aimerais contribuer à ce que l'auditeur/le spectateur se fasse sa propre opinion quant à la réussite ou à l'échec de telle ou telle pièce $e^{10}$.

La liberté d'opinion était en RDA quelque chose de très relatif, voire d'uniquement privé. Dieter Kranz avait su, au fil du temps, se créer un espace où, tout en respectant un cadre idéologique bien défini, il pouvait jouir d'une certaine liberté d'expression. Par ailleurs, il avait réussi à obtenir des syndicats des artistes la permission d'enregistrer les répétitions générales et d'utiliser ce matériel pour ses émissions mensuelles, à raison de vingt minutes d'enregistrements originaux par épisode.

Tandis que Friedrich Luft maintenait la structure très rigide de son émission, toujours réalisée selon le même schéma, Dieter Kranz ouvrait son studio aux différentes professions théatrales, interrogeait souvent les metteurs en scène, les scénographes, les compositeurs et les comédiens. Il semble qu'il ait agi selon son envie et selon la disponibilité de ses interlocuteurs. L'avantage de cette forme est, bien sûr, qu'elle est garante de surprises pour les auditeurs qui, chaque mois, ont droit à une émission leur proposant un concept nouveau. Il n'existe à l'heure actuelle que très peu de documents consacrés à Dieter Kranz, qui est décédé en 2011 à l'âge de 77 ans. Son fils, Oliver Kranz, nous a confié que Dieter Kranz et Friedrich Luft ne s'étaient jamais rencontrés.

Deux cents émissions sont conservées aux Archives nationales de la radio à Potsdam, la moitié d'entre elles environ est consacrée à des opéras, l'autre à des pièces de théâtre. Parmi l'ensemble des titres du programme de Dieter Kranz sur le théâtre, Berlin, Weltstadt des Theaters, que j'ai pu consulter dans ces archives, se dégagent quatre grandes catégories : celles consacrées aux classiques; celles consacrées aux grands auteurs de la RDA, Brecht en particulier; celles consacrées à la vie théâtrale de la RDA - avec ses troupes d'amateurs dans les usines, ses théâtres ouvriers; et, enfin, celles consacrées aux auteurs contemporains venus de ce que l'on appelait le "kapitalistisches Ausland» [les pays capitalistes étrangers], tels Ibsen, Dario Fo, Hans Magnus Enzensberger etc. À titre d'exemple concernant les œuvres classiques, l'une des émissions est consacrée à la mise en scène du Dom Juan de Molière par Benno Besson, au Deutsches Theater. La séquence commence par une prise de son de l'ambiance de la salle, puis on entend les trois coups frappés, dont Dieter Kranz explique à ses auditeurs qu'il s'agit d'une tradition théâtrale française, car cette pratique est inconnue en Allemagne. Intervient alors une musique, et l'émission se poursuit par un entretien avec le metteur en scène suisse qui était venu vivre à Berlin-Est après avoir rencontré Bertolt Brecht, et où il resta jusqu'en 1977.

Poursuivons par les émissions consacrées aux auteurs de la RDA, et notamment à Brecht. En 1978, à l'occasion du $80^{\mathrm{e}}$ anniversaire de la naissance de Brecht, Dieter Kranz diffusa un document qu'il fit qualifier de "sensationnel " par la speakerine qui annonçait l'émission. Effectivement, le cadeau était de taille. Hans Bunge, qui avait été l'assistant de Brecht au Berliner Ensemble mais avait fini par quitter le théatre pour cause de différends avec Helene Weigel, n'était pas en odeur de sainteté auprès des dirigeants de la RDA parce que sympathisant d'opposants au régime. Il avait été congédié en 1965 de l'Académie des arts où il s'occupait des archives de Brecht. Or Dieter Kranz lui donna une heure d'antenne, durant laquelle Hans Bunge diffusa des enregistrements de répétitions du Cercle de craie caucasien et de La Vie de Galilée qu'il avait réalisés en 1954, 1955 et 1956, pour s'assurer de retranscrire fidèlement les indications de mise en scène que Brecht 
souhaitait voir conserver. Pour ce faire, Hans Bunge avait voulu acheter un enregistreur, le Berliner Ensemble refusa de payer et Brecht régla l'achat de sa poche. Brecht était ravi de cet achat et de l'utilisation qu'en faisait Hans Bunge ; le comédien Ernst Busch, en revanche, ne souhaitait pas être enregistré. Hans Bunge rassembla ainsi quarante heures d'enregistrements! Dans l'un des extraits diffusés par Bunge, on entend Brecht lors d'une répétition du Cercle de craie caucasien au Berliner ensemble en 1954 :

Bon, est-ce qu'on va enfin pouvoir commencer? Nous devons essayer de rendre le prologue léger, joyeux, de lui donner du tempo, de l'adoucir dans le sens de « léger et drôle ", n'est-ce pas ? Bien sûr, il y a ces forts mouvements de contestation mais il faut le faire avec de l'humour, n'est-ce pas? On reprend tout avec rapidité et légereté, n'est-ce pas ? Faites-en quelque chose de rapide, sinon cela va devenir une pièce en soi. On recommence ${ }^{11}$.

Imagine-t-on la valeur inestimable de ces enregistrements? Ils réclament à eux seuls une recherche approfondie, qui réserverait sans doute bien des surprises et serait en mesure de remettre en question une certaine doxa concernant la méthode brechtienne !

Lorsque j'ai commencé ces recherches et que j'ai été assez vite confrontée à un relatif manque de clarté quant au corpus qui sommeille dans les archives, je me suis prise à rêver d'une rencontre entre nos deux critiques. Il est sûr qu'il n'y en eut pas. En revanche, j'ai eu la chance de trouver, sur une cassette audio exhumée de l'un des cartons des archives de Dieter Kranz, une rencontre acoustique si l'on peut dire. Il s'agit de deux critiques, l'une par Dieter Kranz, l'autre par Friedrich Luft, de la pièce Coriolan de Shakespeare dans la version de Brecht, jouée à la fin de l'année 1964 au Berliner Ensemble. Nous tenons là la preuve qu'ils ont bien assisté, un soir de décembre 1964, à quelques fauteuils l'un de l'autre, à la première de Coriolan.

Friedrich Luft avait obtenu un visa pour aller à Berlin-Est et commence son émission en abordant la question de la coupure de la ville, dont il ne perd jamais une occasion de se plaindre. Il loue le travail de la troupe, mais finit par porter un jugement sans appel :

La troupe fait preuve de discipline mais elle se distancie tellement de tout qu'à force de distanciation, la sympathie, la persuasion et l'intérêt en finissent presque par mourir. Et ce n'est pas du tout ce que voulait Brecht. Il ne voulait pas que le théâtre se décompose. Mais le danger est grand chez ses épigones ${ }^{12}$.

Dieter Kranz avait quant à lui décidé, après avoir présenté la pièce pendant environ vingt minutes, d'inviter quatre critiques ayant tous publié dans divers journaux un article sur la première de Coriolan, à discuter de l'importance de cette adaptation par Brecht. La conversation porte bien vite sur la nécessité de modifier le texte original pour qu'il puisse être utilisable pour le théâtre socialiste en RDA. Et la question centrale est abordée, celle de la fragilité, de l'inutilité de la figure du « héros ».

Dans toutes les émissions que j'ai écoutées, jamais Dieter Kranz ne se livre à des déclarations d'intention ni à des sermons allant dans le sens de la doctrine prônée par le système. On l'entend laisser ses interlocuteurs aborder les thèmes du «théâtre socialiste », il les écoute, puis résume leurs propos sans que transparaisse son opinion: tout un art. Qui lui a valu, après avoir quitté la radio est-allemande lors de sa dissolution en 1991, de pouvoir continuer à travailler pour les autres radios berlinoises et ouestallemandes, entre autres pour le RIAS, et ensuite pour la Deutschlandradio où je regrette de ne l'avoir jamais rencontré.

Dans son introduction au recueil Théâtre berlinois. 100 mises en scène en trois décennies, publié en 1990, Dieter Kranz ajoute le 5 janvier 1990, juste avant la publication du livre 
qu'il avait pourtant terminé en 1987, une note qui a particulièrement retenu mon attention :

Après l'automne 1989, les trois décennies de théâtre est-berlinois que nous avons documentées apparaissent sous un jour différent. Il est clair maintenant qu'il s'agit d'une époque close, chose qu'on ne pouvait imaginer lorsque nous travaillions à ce livre. Un heureux hasard a voulu que la mise en scène de La Société de transition [de Volker Braun] mette un terme final caractéristique à une évolution du théâtre qui avait commencé de manière aussi significative avec Mère Courage. Les documents de ce livre prouvent que le théâtre sous le régime du " socialisme réellement existant » était tout autre chose qu'un théâtre stalinien. Bien au contraire, on peut y trouver de nombreux éléments prouvant que les artistes de théâtre avaient attiré l'attention sur la déformation des idéaux socialistes et, par là même, sur l'évolution négative qui en découlait. Ce faisant, ils ont contribué à la préparation de ce mouvement populaire qu'ils ont influencé de façon déterminante pendant l'automne $1989^{13}$.

En guise de conclusion, j'aimerais souligner la richesse prodigieuse et encore non explorée des Archives nationales de la radio et des Archives de l'Académie des arts. Je n'ai pas parlé de la censure qui, officiellement, n'existait pas, mais elle est, à l'est comme à l'ouest d'ailleurs, inhérente aux choix qu'ont fait ces critiques et elle mériterait qu'on s'y intéresse. Friedrich Luft et Dieter Kranz représentaient deux types de critiques de théâtre fort différents et cette différence est sans doute liée aux systèmes dans lesquels ils vivaient. Mais ils n'étaient pas les seuls, d'autres critiques tels que Joachim Werner Preuß et Dieter Steinbeck ont eux aussi, des dizaines d'années durant, critiqué les pièces jouées à Berlin pour la station de radio berlinoise SFB (Sender Freies Berlin, Station Berlin Libre). Pendant des dizaines d'années, tout Berlinois s'intéressant au théâtre pouvait ainsi écouter, le dimanche matin, de dix heures et demie à midi en changeant deux fois de programme, trois émissions se rapportant au monde du théâtre dans sa ville ${ }^{14}$. De cette période révolue témoignent des kilomètres de bandes magnétiques qui font revivre à ceux qui les écoutent un monde disparu mais acoustiquement bien vivant! Pour notre plus grand plaisir auditif.

\section{BIBLIOGRAPHIE}

Agde, Günter, critique du livre de Günter Holzweißig, Die schärfste Waffe der Partei : Eine

Mediengeschichte der DDR [L'arme la plus puissante du Parti, histoire des médias en RDA], publiée sur H-Soz-u-Kult, plateforme d'information et de communication pour historiens en ligne, juin 2003.

Hermann, Ingo (dir.), Friedrich Luft, Stimme der Kritik, Zeugen der Jahrhundert, Göttingen, Lamuv, 1991.

Kohse, Petra, Gleiche Welle, gleiche Stelle, Berlin, Aufbau Verlag, 1998.

Kranz, Dieter, Berliner Theater. 100 Aufführungen aus drei Jahrzehnten [Théâtre berlinois. 100 mises en scène en 3 décennies], Berlin, Henschel Verlag, 1990. 
Luft, Friedrich, Stimme der Kritik. Theaterereignisse seit 1965 [Voix de la critique. Évènements dramatiques depuis 1965], Stuttgart, Deutsche Verlags-Anstalt, BRD, 1979.

\section{NOTES}

1. Entretien de Friedrich Luft avec Horst Eifler, «Kritische Jahre », diffusé le 12 mars 1981 par le RIAS et cité par P. Kohse, Gleiche Welle, gleiche Stelle, p. 46.

2. Cité dans F. Luft, Stimme der Kritik, p. 59.

3. Id.

4. "Luft ist mein Name, Friedrich Luft. Ich bin 1,86 groß, dunkelblond, wiege 122 Pfund, habe Deutsch, Englisch, Geschichte und Kunst studiert, bin geboren im Jahre 1911, bin theaterbesessen und kinofreudig und beziehe die Lebensmittel der Stufe II. Zu allem trage ich neben dem letzten Anzug, den ich aus dem Krieg gerettet habe, eine Hornbrille auf der Nase. Wozu bin ich da? - Ich soll mich für Sie plagen ». (Je traduis.)

5. Cité dans F. Luft, Stimme der Kritik, p. 56.

6. Ibid., p. 57.

7. Manuscrit non publié de l'émission Stimme der Kritik de F. Luft, en date du 20 avril 1946 (Friedrich-Luft-Archiv, Akademie der Künste, Robert-Koch-Platz, Berlin).

8. Il ne m'a pas été possible de retrouver la source de cette citation capitale pour définir le travail de critique, tel que le concevait Friedrich Luft. Elle semble provenir de l'une de ses émissions et avoir été relayée par des articles qui lui ont été consacrés par la suite.

9. Je traduis.

10. Cité dans l'hommage rendu à Dieter Kranz sur la page internet du Preis der Schallplattenkritik.

11. Je traduis.

12. F. Luft, émission Stimme der Kritik du 27 décembre 1964, RIAS Berlin. Transcription par C. Cossais d'une cassette audio conservée aux archives Kranz de l'Académie des arts de Berlin, Robert-Koch-Platz.

13. D. Kranz, Berliner Theater. 100 Aufführungen aus drei Jahrzehnten, p. 10.

14. Cf. la critique par G. Agde du livre de Günter Holzweißig, Die schärfste Waffe der Partei: Eine Mediengeschichte der DDR.

\section{RÉSUMÉS}

«Le théâtre chante, nous nous en faisons l'écho ». De 1946 à 1990, le critique Friedrich Luft partagea sa passion du théâtre avec les auditeurs de la station de radio ouest-berlinoise RIAS, en animant hebdomadairement une émission de quinze minutes, Stimme der Kritik [Voix de la critique], dans laquelle il présentait les mises en scène qui lui avaient plu. Outre ce format, il avait également conçu en 1951 une émission mensuelle d'environ trois quarts d'heure, Wir gehen ins Theater [Nous allons au théâtre], qui faisait entendre des extraits de pièces du répertoire berlinois de l'époque. De l'autre côté de la frontière, une dizaine d'années après, un autre critique de théâtre, Dieter Kranz, avait créé à Berlin-Est une émission symétrique de quinze minutes Atelier und Bühne [Atelier et Scène] qui fut diffusée de 1956 à 1991. Il dirigea ensuite, de 1961 à 
1991, une émission mensuelle de quarante-cinq minutes, Berlin - Weltstadt des Theaters [Berlin, capitale mondiale du théâtre], dans laquelle, ayant obtenu des théâtres le droit d'enregistrer les répétitions générales, il introduisait des interviews avec l'auteur, le metteur en scène, le scénographe ou certains des comédiens. De ces œuvres radiophoniques restent des centaines d'heures de pur plaisir acoustique qui donnent un aperçu étonnant du théâtre dans le Berlin d'après-guerre. Le présent article révèle l'activité de ces deux critiques de théâtre berlinois et les resitue dans leur contexte historique et politique.

"The theatre sings; we echo it ». From 1946 to 1990, the critic Friedrich Luft shared his passion for the theatre with the listeners of the West Berlin radio station RIAS in presenting a weekly fifteen-minute broadcast, Stimme der Kritik [Voices of criticism] during which he introduced to the productions which had appealed to him. Beyond this format, in 1951, he had also conceived a monthly broadcast of about forty-five minutes, Wir gehen ins Theater [We go to the theatre] in which excerpts from the Berlin repertoire of the time were heard. Across the border, some ten years later in East Berlin, another theatre critic, Dieter Kranz, had created a symmetrical fifteenminute broadcast, Atelier und Bühne [Workshop and stage], which was heard from 1956 to 1991. Then, from 1961 to 1991 he managed a monthly forty-five-minute broadcast, Berlin-Weltstadt des Theaters [Berlin - world capital of the theatre] during which, having obtained from the theatres the right to record dress rehearsals, he introduced to interviews with the playwright, the producer, the scenographer, or some of the comedians. Of all these radio works are left hundreds of hours of pure acoustic pleasure which leave a stunning account of the theatre in postwar Berlin. The present article reveals these two Berlin theatre critics' activities and put them back in their historical and political context.

INDEX

Mots-clés : Allemagne, Berlin, critiques de théâtre, radio, Friedrich Luft, Dieter Kranz, aprèsguerre, RIAS, Berliner Rundfunk

Keywords : Germany, Berlin, theatre critics, radio, Friedrich Luft, Dieter Kranz, postwar, RIAS, Berlin radio

\section{AUTEUR}

\section{CLARISSE COSSAIS}

Docteur en civilisation germanique, auteur, réalisatrice et productrice à la radio publique Deutschlandradio Kultur.

Parmi ses réalisations radiophoniques en tant qu'auteur :

« Ich denke die Poesie dreidimensional », portrait de Michèle Métail - poète et artiste, émission de 30 minutes diffusée le 30 juil. 2013 sur Deutschlandradio Kultur : http:// www.deutschlandradiokultur.de/ich-denke-die-poesie-dreidimensional.974.de.html? dram:article_id=255181

«Vergangenes neu schreiben, Unbekanntes zähmen », Französische Schriftsteller der 3. Generation schreiben über den 2. Weltkrieg, émission de 30 minutes diffusée le 23 janv. 2015 sur Deutschlandradio Kultur : http://www.deutschlandradiokultur.de/franzoesische-schriftstellerdritte-generation-schreibt.976.de.html?dram:article_id=309557

« Histoire de R - Geschichte eines Lauts », émission de 54'21“ sur le « $\mathrm{r}$ » roulé en français, 
diffusée le 4 juin 2016 sur Deutschlandradio Kultur : http://www.deutschlandradiokultur.de/ reihe-neues-aus-der-provinz-histoire-de r.958.de.html ?dram :article_id =350317 